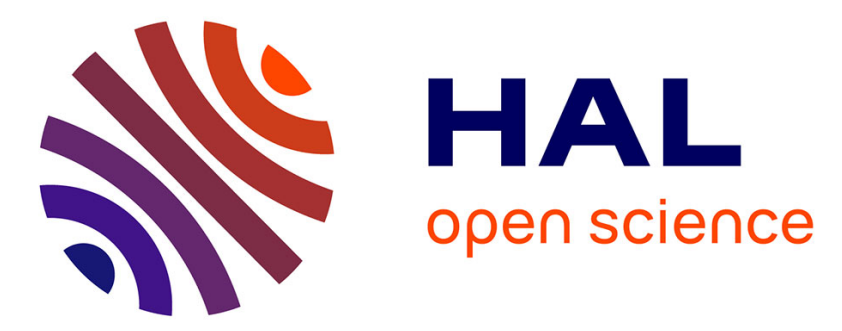

\title{
Low-Frequency Behavior of Beads Constrained on a Lattice
}

Bruno Gilles, Christophe Coste

\section{To cite this version:}

Bruno Gilles, Christophe Coste. Low-Frequency Behavior of Beads Constrained on a Lattice. Physical Review Letters, 2003, 90 (17), pp.174302. 10.1103/PhysRevLett.90.174302 . hal-01406751

\section{HAL Id: hal-01406751 \\ https://hal-univ-paris.archives-ouvertes.fr/hal-01406751}

Submitted on 1 Dec 2016

HAL is a multi-disciplinary open access archive for the deposit and dissemination of scientific research documents, whether they are published or not. The documents may come from teaching and research institutions in France or abroad, or from public or private research centers.
L'archive ouverte pluridisciplinaire $\mathbf{H A L}$, est destinée au dépôt et à la diffusion de documents scientifiques de niveau recherche, publiés ou non, émanant des établissements d'enseignement et de recherche français ou étrangers, des laboratoires publics ou privés.

\section{(c)(1)}

Distributed under a Creative Commons Attribution| 4.0 International License 


\title{
Low-Frequency Behavior of Beads Constrained on a Lattice
}

\author{
Bruno Gilles \\ Laboratoire de Physique, ENS Lyon, 46 Allée d'Italie, 69364 Lyon Cedex 07, France \\ Christophe Coste \\ Groupe de Physique des Solides, 2 place Jussieu, 75251 Paris Cedex 05, France
}

(Received 8 January 2003; published 2 May 2003)

\begin{abstract}
We study sound propagation in a triangular lattice of spherical beads under isotropic stress. Polydispersity of real beads breaks some contacts, creating a disordered lattice of contacting beads. At large stress, the sound velocity behaves according to Hertz contact law and departs from it at lower stress. This evolution is reversible, with the same crossover when increasing or decreasing the stress, for a given piling. Correlations are much more sensitive to disorder. When calculated with signals propagated in the same lattice, they evolve reversibly with the stress, being much higher at large stress when the contact lattice is more regular. This leads to an interpretation of the non-Hertzian behavior in terms of progressive activation of contacts, in discrepancy with previous models involving buckling of force chains.
\end{abstract}

DOI: 10.1103/PhysRevLett.90.174302

A prominent feature of granular media is the heterogeneity of the spatial distribution of forces in the piling, observed both in simulations [1,2] and experiments [3]. A lattice of contacting grains supports the stresses and allows acoustic wave propagation. Even for the simplest model of granular media, that is, nominally monodisperse spherical beads whose centers are placed on a regular lattice, the geometry of the contact lattice may be extremely complicated. Indeed, since spherical grains have only point contacts, any imperfections of the beads imply that some contacts are broken, creating randomness.

This effect has been set forth to explain the paradoxical propagation of sound in a face-centered cubic lattice of steel beads [4,5]: Hertz contact law [6] predicts that the sound velocity should scale as the power $1 / 6$ of the force applied to the piling, whereas a $1 / 4$ exponent is observed. Some authors $[5,7,8]$ suggested that actual beads cannot be considered as perfect elastic spheres. They proposed modifications of Hertz law that led to $1 / 4$ exponent. Another approach [1,5,9] attributes to randomness of the contact lattice the discrepancy with Hertz law predictions.

In a previous work [10], we tested the validity of the first approach on a chain of identical beads, in which all the grains are in contact with their neighbors. Our measurements show Hertzian behavior, which rules out this approach. In this Letter, we report on experiments done with a $2 \mathrm{D}$ system, which is adequate to study the consequences of the randomness of the contact lattice.

The experimental setup is sketched in Fig. 1. The bead centers are placed on a regular triangular lattice, contained in a hexagonal cell. The lattice is horizontal, held between two plates of polytetrafluoroethylene, in order to get low friction and poor impedance matching between the lattice and the framework [10]. Three sides of the
PACS numbers: 45.70.-n, 43.20.+g, 46.65.+g

hexagon are fixed, while the other ones may move independently along their normal direction. The force they exert on the lattice is measured by a static force sensor. With the help of a feedback loop, we may apply a stress at any prescribed value. In the following, the stress is applied isotropically.

We use either stainless steel beads of diameter $d=$ $8 \mathrm{~mm} \pm 4 \mu \mathrm{m}$ or nylon beads $(d=8 \mathrm{~mm} \pm 50 \mu \mathrm{m})$. An important feature of our experiment is that both the acoustic transmitter and the sensor are in contact with only one bead. This configuration is particularly sensible to disorder [11].

A carefully designed acoustic transmitter $[10,12]$ allows us to send a burst wave of well defined frequency $6.5 \mathrm{kHz}$, with a Gaussian envelope. As shown by Fig. 2, curves $(a)$ and $(b)$, the acoustic signal closely follows the

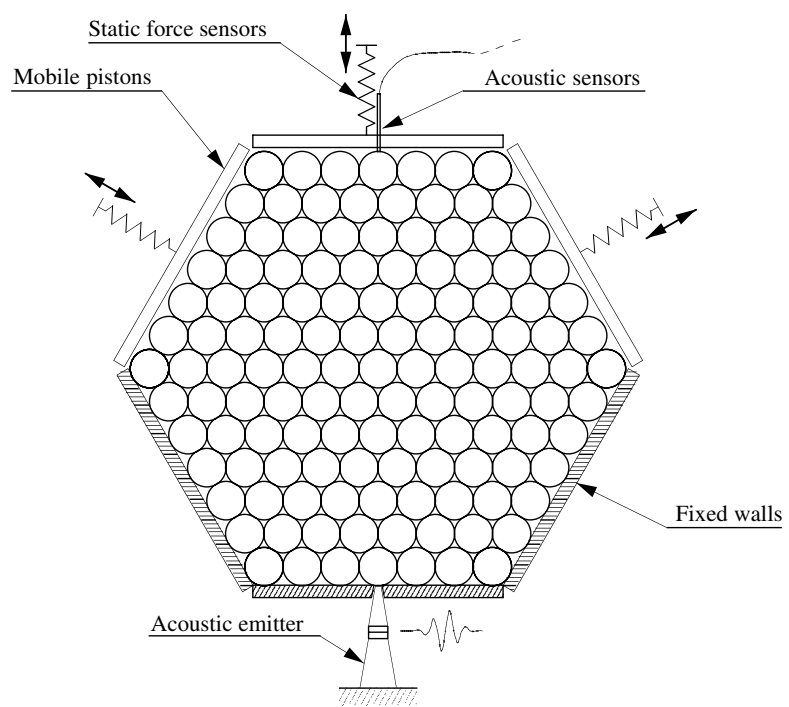

FIG. 1. Sketch of the experimental setup. 


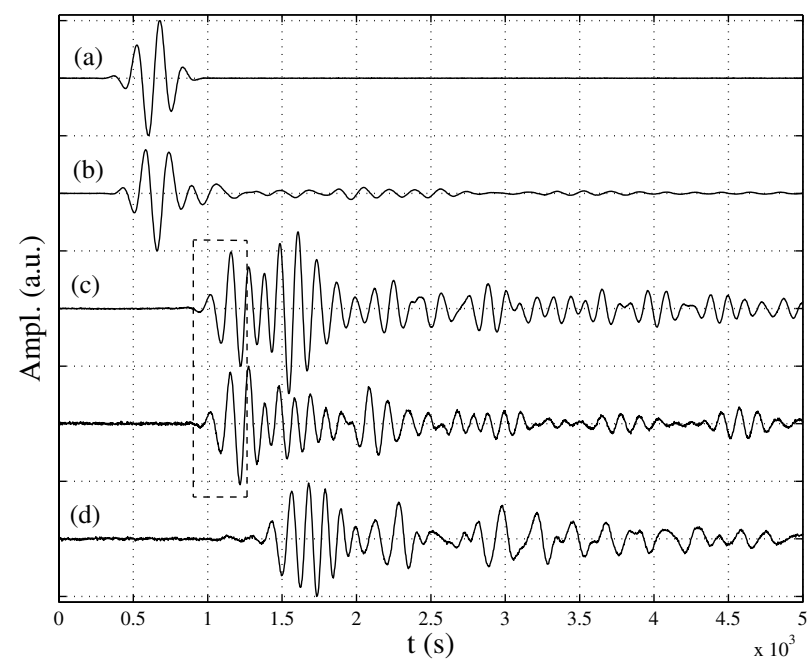

FIG. 2. Structure of the signal. Curves: $(a)$ electrical excitation; (b) acoustical response; (c) signals propagated in two different steel bead lattices. The box delimits the coherent signal. Curve $(d)$ shows the signal propagated in nylon beads.

electrical excitation. We measure the time of flight (TOF) of the wave. As we shall see, the arrival of the signal corresponds to the propagation of a coherent wave in an effective medium, so that we can estimate a velocity from the distance between transmitter and sensor. The TOF varies between 500 and $1000 \mu$ s, depending on the applied force (see Fig. 3) in steel beads, and between 900 and $1400 \mu$ s (see Fig. 4) in nylon beads. Thus the typical wavelength ranges from 8 to 16 (respectively, 6 to 9) bead diameters $d$, for steel (respectively, nylon). The lattice side $L$ is 31 beads, so that the wavelength is intermediate between the two natural length scales $L$ and $d$ of the system, which is consistent with the notion of an effective medium.

Each experimental run proceeds in the same way: first, we arrange the beads with their centers on a triangular lattice, thus getting a peculiar (but unknown) initial distribution of bead diameters in the lattice. Then we increase the static force applied on the lattice, without making any measurement: since the lattice holds the stress, the contact network is established. It evolves progressively with the applied force through mechanisms we will detail further. At the end of this first compression, we get a peculiar realization of the contact lattice. Then, we proceed to the measurements by slowly decreasing the force, each decrement being roughly $10 \%$ in magnitude. Having reached the minimal force (below which we are no more able to collect the acoustical signal), we may increase it subsequently in the same way.

From Fig. 2, curve (c), it is clear that the acoustical signal is strongly distorted after propagation in steel beads. The tail of the signal is partly due to reflections of the waves on the boundaries and partly to multiple scattering by the disordered contact lattice. Wave amplitude is small enough to keep the lattice undisturbed, so that it does not affect the shape of the signal which is

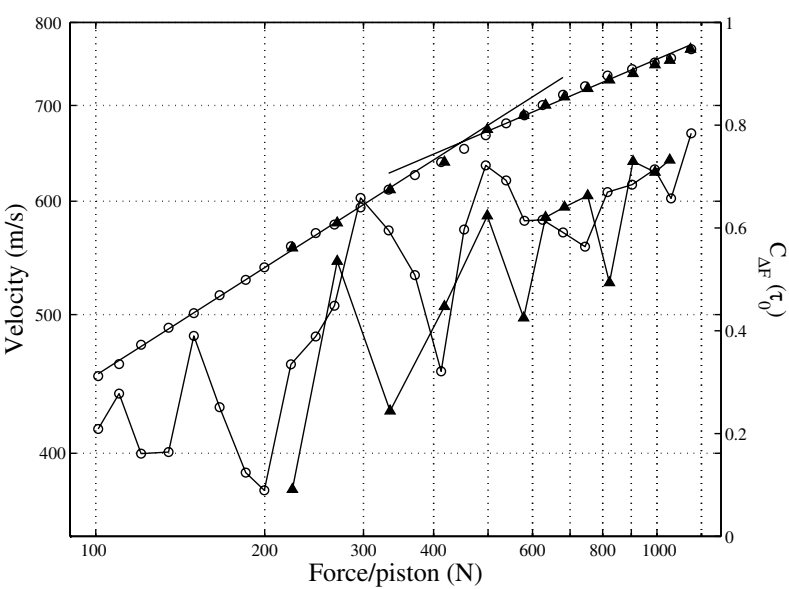

FIG. 3. Left ordinate: plot, in log-log scale, of the velocity versus the applied force, in steel beads, during compression $(O)$ and decompression $(\boldsymbol{\Lambda})$ of the same lattice. Right ordinate: plot, in log-linear scale, of the correlation $C_{\Delta F}\left(\tau_{0}\right)$ [cf. Equation (3)] (same symbols, joined by a solid line).

perfectly reproducible under constant stress. This is not so when we consider two such measurements for two different initial random mixing of the beads: In that case, the two contact lattices are not the same. As shown in Fig. 2, curve $(c)$, the first oscillations are reproducible, but the tails are completely different, which is an evidence that the wave propagation depends on the details of the contact lattice. We identify those first, reproducible, oscillations as the coherent signal [11] and the tail as the incoherent signal [13].

For the coherent part of the wave, which propagates in an effective medium, we may define a velocity as the ratio of emittor-sensor distance and the TOF. Its evolution with the static force applied to the piling is shown in Fig. 3. Assuming perfect ordering of the contact lattice, Hertz law predicts a power $1 / 6$ scaling with the force [4].

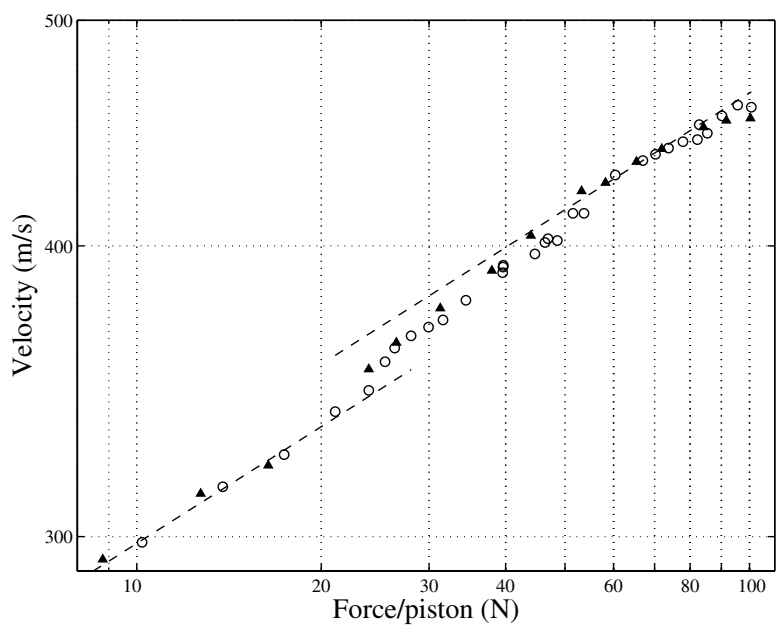

FIG. 4. Plot, in log-log scale, of the velocity versus the force, in nylon beads, during compression $(\bigcirc)$ and decompression $(\boldsymbol{A})$ of the same lattice. 
At moderate force we observe a non-Hertzian exponent [14], followed by a Hertzian one at larger force. What is remarkable is that we observe the same exponent as in $3 \mathrm{D}$ systems, roughly $1 / 4[4,11]$. This exponent is thus almost insensitive to the piling dimensionality. The two regimes are always observed with steel beads. The high stress Hertzian regime is very reproducible, with a scaling for the velocity $c_{s}=(255 \pm 5) F_{\text {stat }}^{1 / 6} \mathrm{~m} / \mathrm{s}$ (with $F_{\text {stat }}$ in $N$ ). The prefactor is in very good agreement with the expected value of $261 \mathrm{~m} / \mathrm{s} / \mathrm{N}^{-1 / 6}$ for a triangular lattice [16]. This is an evidence that, at high stress, the contact lattice becomes more and more homogeneous. In contrast, the crossover between the two regimes strongly depends on the initial lattice and is measured between 190 and $325 \mathrm{~N}$. The amplitude of velocity fluctuations in the nonHertzian regime, at a given force for different initial lattices, is about $20 \%$, which is rather small. It illustrates the self-averaging aspect of effective properties such as sound velocity. As we see below, properties of the incoherent part of the signal such as time correlations may fluctuate much more strongly.

The non-Hertzian regime reflects the evolution of the contacting grain lattice under compression. Two mechanisms have been put forward to explain this evolution, either successive buckling instabilities of force chains [5] or progressive activation of contacts by elastic deformation of the beads [1]. Buckling occurs only under increasing stress and implies a highly irreversible behavior of the lattice. On the contrary, contact activation is reversible since it relies on the elasticity of the beads. As shown in Fig. 3, the velocity takes the same values during compression or decompression, and most importantly the crossover between Hertzian and non-Hertzian regimes is the same, for a given initial realization of the lattice. This reversible behavior is an evidence that the lattice evolves via activation/deactivation of contacts. Under increasing force contacts are progressively activated, leading to a ramification of the contact lattice, and the effective stiffness of this structure increases more quickly than that of individual contacts. The sound velocity exponent is thus greater than expected from Hertz law. At very large force, almost all grains are in contact and we recover a quasiregular contact lattice and its Hertzian signature.

As evidenced by Fig. 4, the behavior of nylon bead lattice is somewhat different. We observe a new, low force Hertzian behavior. For a given lattice, the evolution of sound velocity is still reversible: velocity values and crossover positions are the same under compression and decompression, even in the new low force regime.

From the Hertz solution [6], one can roughly estimate the ratios of the distances of approach $\delta$ for steel and nylon beads,

$$
\frac{\delta_{\text {nylon }}}{\delta_{\text {steel }}}=\left(\frac{E_{\text {steel }}}{E_{\text {nylon }}} \frac{F_{\text {nylon }}}{F_{\text {steel }}}\right)^{2 / 3} \approx 4
$$

where $F_{\text {steel }}$ (respectively, $F_{\text {nylon }}$ ) is the midscale force taken from Fig. 3 (respectively, Fig. 4 ), and $E$ is the Young's modulus $\left(E=2.26 \times 10^{11} \mathrm{~N} / \mathrm{m}^{2}\right.$ for steel and $E=3.55 \times 10^{9} \mathrm{~N} / \mathrm{m}^{2}$ for nylon). Thus the increase of the distance of approach for nylon beads is insufficient to compensate the factor 10 in radius dispersion. Hence the initial tenuous structure which holds the stress under very low compression is much more stable in nylon than in steel beads. Under increasing stress, it first evolves elastically, without much new contacts, causing this low force Hertzian regime. Then, more contacts are activated and we recover the non-Hertzian regime already observed with steel beads.

As with steel beads, the high stress Hertzian regime is very reproducible, with an experimental prefactor $227 \pm$ $5 \mathrm{~m} / \mathrm{s} / \mathrm{N}^{-1 / 6}$ in very good agreement with the calculated one $233 \mathrm{~m} / \mathrm{s} / \mathrm{N}^{-1 / 6}$. The crossover positions strongly depend on the initial lattice, ranging, respectively, from 15 to $25 \mathrm{~N}$ and from 50 to $60 \mathrm{~N}$.

A low force Hertzian behavior has been predicted by Roux [1] in simulations of our experimental system. He observed it for $\frac{2 P}{\alpha^{3 / 2} E d}<10^{-3}$, where $P$ is the $2 \mathrm{D}$ pressure and $\alpha$ is the relative dispersion in diameter. This gives an estimate of, respectively, $7 \mathrm{~N}$ (for steel) and $2 \mathrm{~N}$ (for nylon) as the crossover between low force Hertzian and non-Hertzian behaviors. Our measurements are in qualitative agreement with this prediction, since we go down to much lower forces with nylon beads than with steel beads. But the corresponding crossover observed with nylon bead lattice is $20 \mathrm{~N}$. A possible explanation of this discrepancy is friction between grains, not taken into account in the simulations.

Recently, a new theoretical approach [15] has been used to calculate the effective speed of sound in the same system. This mean field calculation, valid in the high force limit where force fluctuations are not too strong, takes into account the evolution of the number of effective contacts with the pressure exerted on the lattice. The sound velocity as a function of the pressure agrees very well with our data and the simulations of Roux [1], at moderate or high pressure. As emphasized in [14], this analytical solution cannot be simply writen as a power law.

To compare signal shapes, particularly sensitive to disorder, we use cross correlations. If each signal consists in $N$ samples $s_{i}\left(t_{n}\right)$ taken at times $t_{n}$, it reads

$$
C^{1,2} \equiv \frac{\sum_{n=1}^{N} s_{1}\left(t_{n}\right) s_{2}\left(t_{n}\right)}{\sqrt{\sum_{n=1}^{N} s_{1}^{2}\left(t_{n}\right)} \sqrt{\sum_{n=1}^{N} s_{2}^{2}\left(t_{n}\right)}} .
$$

We restrict this calculation either to the coherent signal, denoting $C_{\text {coh }}$, or to the acoustic speckle, denoting $C_{\text {incoh }}$. The correlations are calculated at the same static force, either for the same lattice under compression and decompression $\left(C^{\simeq}\right)$ or for two lattices with different initial preparation $\left(C^{\neq}\right)$. 


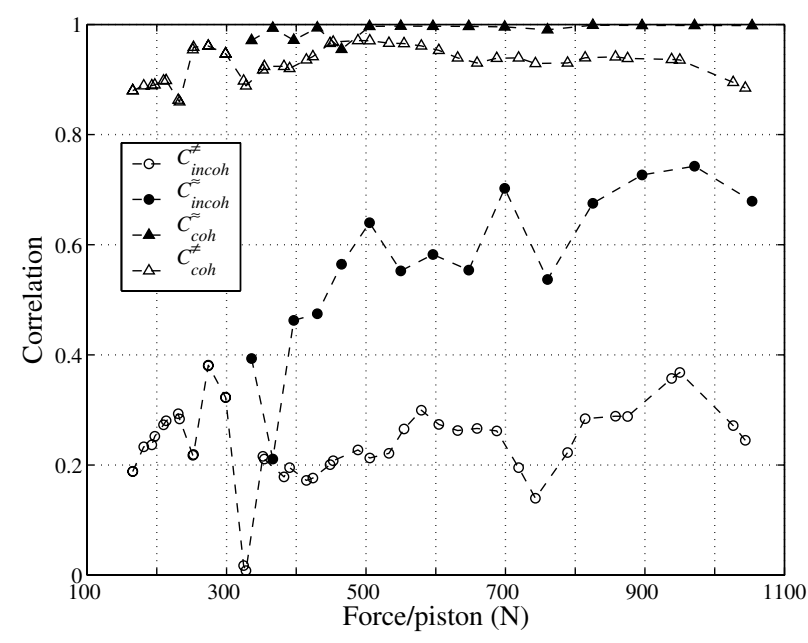

FIG. 5. Correlations versus the force, in a steel beads lattice, for compression/decompression of the same lattice $C^{\simeq}(\mathbf{O})$, and for two different contact lattices, $C^{\neq}(\bigcirc)$. [See Eq. (2)].

The results are summarized in Fig. 5. The correlations $C_{\text {coh }}^{\simeq}$ and $C_{\text {coh }}^{\neq}$are always greater than 0.8 . This is consistent with the concept of coherent signal, whose properties depend only on the effective propagation medium, not on the details of the contact lattice. In contrast, the correlations $C_{\text {incoh }}^{\simeq}$ and $C_{\text {incoh }}^{\neq}$are smaller, and we always have $C_{\text {incoh }}^{\neq}<C_{\text {incoh }}^{\simeq}$. For lattices with different initial statistical distributions of beads, $C_{\text {incoh }}^{\neq}$is small. It is remarkable that this effect persists far away in the Hertzian regime, showing that correlations are a much more sensitive probe than velocity measurements and that some disorder remains even with high applied forces. On the contrary, waves propagating during compression and decompression of the same lattice are highly correlated. It is another evidence of reversibility and that buckling of force chains is not the relevant phenomenon for the evolution of the contact lattice.

In our confined setup, the tail of the signal originates both from multiple scattering in a disordered medium and from multiple reflections on the boundaries. Since reflection holds on fixed boundaries and involves mostly the coherent signal, it should not depend much on the details of the lattice. On the contrary, the weak values of $C_{\text {incoh }}^{\neq}$ (see Fig. 5) show that the incoherent signal highly depends on lattice configuration. That means that multiple scattering is predominantly responsible for its structure and justifies a posteriori to call it incoherent.

To describe the contact lattice evolution with the force, we calculate the correlation for two successive forces $F$ and $F+\Delta F$, during a monotonic evolution of the stress,

$$
C_{\Delta F}\left(\tau_{0}\right) \equiv \frac{\sum_{n=1}^{N} s_{F}\left(t_{n}\right) s_{F+\Delta F}\left(t_{n}+\tau_{0}\right)}{\sqrt{\sum_{n=1}^{N} s_{F}^{2}\left(t_{n}\right)} \sqrt{\sum_{n=1}^{N} s_{F+\Delta F}^{2}\left(t_{n}\right)}} .
$$

Here $\tau_{0}$ is the delay caused by the sound velocity variation due to the force increment $\Delta F$. As shown by Fig. 3, the correlation is much higher in the high force Hertzian regime, which supports our previous interpretation: at large force, almost all contacts are active, the lattice evolves very little under a force increment, and the correlation should be high. On the contrary, it is small in the non-Hertzian regime where the lattice evolves a lot by progressive activation of contacts. The reproducibility of the correlation under compression and decompression indicates that the contact lattice evolves reversibly.

We have shown that a regular lattice of nominally identical beads actually behaves as a disordered system. The sound velocity dependency on the stress is steeper than what may be expected from Hertzian behavior of a perfect lattice [4]. This is due to structural modification of the contact lattice, via progressive activation of contacts. The reversibility of this process is evidenced by the lack of hysteresis in both velocity and correlation measurements during compression/decompression of the system. This is presumably true for more general granular media.

We acknowledge useful discussions with C. Caroli and B. Velicky during the completion of this work. We thank D. Bouraya for technical help in building the setup.

[1] J.-N. Roux, in Powders and Grains 97, edited by R. P. Behringer and J. T. Jenkins (Balkema, Rotterdam, 1997).

[2] F. Radjai, M. Jean, J.-J. Moreau, and S. Roux, Phys. Rev. Lett. 77, 274 (1996).

[3] T. Travers, M. Ammi, D. Bideau, A. Gervois, J.-C. Messager, and J.-P. Troadec, J. Phys. (Paris) 49, 939 (1988).

[4] J. Duffy and R. D. Mindlin, J. Appl. Mech. 24, 585 (1957).

[5] J. D. Goddard, Proc. R. Soc. London A 430, 105 (1990).

[6] L. D. Landau and E. Lifshitz, Theory of Elasticity (Pergamon, Oxford, 1986).

[7] P.-G. de Gennes (unpublished).

[8] P.-G. de Gennes, Europhys. Lett. 35, 145 (1996).

[9] H. J. Hermann, D. Stauffer, and S. Roux, Europhys. Lett. 3, 265 (1987).

[10] C. Coste and B. Gilles, Eur. Phys. J. B 7, 155 (1999).

[11] X. Jia, C. Caroli, and B. Velicky, Phys. Rev. Lett. 82, 1863 (1999).

[12] A. Bangviwat, H. K. Ponnekanti, and R. D. Finch, J. Acoust. Soc. Am. 90, 1223 (1991).

[13] This is not obvious, and discussed at length below.

[14] We follow what is customary in the literature. There is no fundamental reason for the velocity to follow a power law in non-Hertzian regimes, and some evidence that it does not [15].

[15] B. Velicky and C. Caroli, Phys. Rev. E 65, 021307 (2002).

[16] E. J. Garboczi and M. F. Thorpe, Phys. Rev. B 32, 4513 (1985). 